\title{
DIFERENCIAS DE GÉNERO Y RENDIMIENTO EN ESTUDIANTES DE EDUCACIÓN INFANTIL DE LA UNIVERSIDAD SURCOLOMBIANA
}

\section{GENDER DIFFERENCES AND PERFORMANCE IN EARIY CHILDHOOD EDUCATION STUDENTS AT THE UNIVERSIDAD SURCOLOMBIANA}

\section{Gloria Chávarro Medina ${ }^{1}$}

\section{Alix Casadiego Cabrales ${ }^{2}$}

\section{Leidy Carolina Cuervo ${ }^{3}$}

\section{Álvaro Avendaño Rodríguez ${ }^{4}$,}

Universidad Surcolombiana, Neiva $(\mathrm{H})$, Colombia

\footnotetext{
$1 \quad$ Licenciada en Pedagogía Infantil- Universidad Surcolombiana, Mg. en educación Universidad Surcolombiana. ORCID https://orcid.org/0000-0002-0074-0530

gloria.chavarro@usco.edu.co, tel. (057) 8754753, Universidad Surcolombiana, Neiva (H), Colombia

2 Licenciada en Pedagogía Infantil- Universidad Surcolombiana, Mg. en educación Universidad Surcolombiana, Doctora en Sociedad del Conocimiento, Universidad Internacional de la Rioja. ORCID http://orcid.org/0000-0003-4376-5874 alix.casadiego@usco.edu.co, tel. (057) 8754753, Universidad Surcolombiana, Neiva (H), Colombia

3 Licenciada en Pedagogía Infantil- Universidad Surcolombiana, Mg. en educación Universidad Surcolombiana, Doctora en Investigación y Docencia-UNADE-México, Vicerrectora de Investigación y Proyección ORCID https://orcid.org/0000-00026927-0776 carolina.cuervo@usco.edu.co, tel. (057) 8645141Universidad Surcolombiana, Neiva (H), Colombia

$4 \quad$ Mg. Desarrollo Comunitario, Universidad Surcolombiana, Docente de planta, Universidad Surcolombiana

ORCID https://orcid.org/0000-0003-0300-400X alave@usco.edu.co, tel. (057) 8754753 Universidad Surcolombiana, Neiva (H), Colombia
} 


\section{RESUMEN}

El presente estudio tiene como finalidad establecer si el género es un factor significativo en el rendimiento de los estudiantes en Educación Infantil. Para tal fin, se obtuvieron los promedios de calificaciones de 667 estudiantes de Educación Infantil de la Facultad de Educación de la Universidad Surcolombiana (658 mujeres y 9 hombres), del año 2013 al 2018. En general, los resultados muestran que no hay diferencias estadísticamente significativas. Se concluye que la variable género no influye en el rendimiento de los cursos de Educación infantil.

PALABRAS CLAVE: Educación Infantil, género, hombres, mujeres, carrera universitaria.

SUMMARY: The purpose of this study is to establish if gender is a significant factor in the performance of students in Early Childhood Education. For this purpose, the grade averages of 667 Early Childhood Education students from the Faculty of Education of the Universidad Surcolombiana (658 women and 9 men) were obtained, from 2013 to 2018 . In general, the results show that there are no statistically differences. significant. It is concluded that the gender variable does not influence the performance of infant education courses.

KEY WORDS: Early childhood education, gender, men, women, university degree.

\section{INTRODUCCIÓN}

La selección de la carrera universitaria define en gran parte el futuro laboral de los graduados, sin embargo, las diferencias de género juegan un papel fundamental en la toma de estas decisiones. Existen grandes diferencias de género en el mercado laboral, las mujeres enfrentan barreras más altas para ingresar a los sectores de manufactura, construcción y transporte, y los hombres enfrentan barreras más altas para ingresar al sector de servicios; las universidades desempeñan un papel destacado que parece reproducir las diferencias de género en lugar de nivelarlas, (Cozzi et al, 2018). Los hombres comienzan sus carreras desde los niveles más altos de la jerarquía que las mujeres, pero las diferencias de género en las elecciones educativas, explican gran parte de esta brecha, (Kauhanen \& Napari, 2011).

Skachkova (2007), presenta las voces de profesoras inmigrantes en Estados Unidos sobre el clima social que prevalece en sus instituciones que afecta sus carreras académicas; para el autor, estos desafíos políticos al etnocentrismo, el sexismo y el racismo en la academia, podría provenir de una mayor internalización del sistema estadounidense de educación superior. Se necesita un esfuerzo nacional sistemático individual, niveles institucionales y gubernamentales para cambiar el espíritu de miedo a eso de abrazar a los inmigrantes (Cole 2003, citado en Skachkova, 2007).

Existen factores que tienen un efecto en las oportunidades de las mujeres para carreras universitarias, como la incompatibilidad de roles profesionales y familiares y los mecanismos de discriminación invisible; la familia es una responsabilidad, pero también un recurso para la mujer, las mayores tasas de productividad de los investigadores casados, tanto mujeres como hombres, y los datos sobre el uso del tiempo indican que, como tal, las responsabilidades familiares no son incompatibles con una investigación y una carrera universitaria, (Luukkonen-Gronow, 1987). En el entorno de las teorías tradicionales, se construyen las afirmaciones cotidianas que expresan los juicios de valor que determinan a cada uno de los sexos, y que se transmiten por la vía familiar y la comunidad; estos valores se van infiltrando en la vida de las personas, desde la educación de los más pequeños, y esto consagra las situaciones de diferencia delimitadas sexualmente, (Apple, 1989 citado en Albertín \& Zufiaurre, 2005). 
La educación del padre es particularmente importante para los hijos, los niños se benefician más de un entorno familiar ventajoso que las niñas en términos de resultados de la escuela primaria, sin embargo, las brechas de género en el nivel educativo, el empleo y los ingresos están aumentando en la educación materna, beneficiando a las hijas (Brenøe \& Lundberg, 2018).

El matrimonio y los hijos continúan afectando las opciones de carrera de las mujeres profesionales más que las de los hombres y las actividades profesionales parecen estresar los matrimonios de las mujeres y limitar las oportunidades personales para las mujeres más que para los hombres, las mujeres como grupo aún encuentran obstáculos para sus carreras profesionales y limitaciones en sus vidas personales que los hombres no enfrentan, (Burton \& Darcy, 1985). En este sentido, (Ferber \& Huber, 1979) examina hasta qué punto el nivel de educación del cónyuge obstaculiza o ayuda a las carreras de doctorado, encontrando que tener un cónyuge con estudios de doctorado, afectó negativamente la participación de las esposas en la fuerza laboral tanto para los esposos como para las esposas, el matrimonio con un cónyuge altamente educado tiene algunos efectos negativos en la carrera.

En las carreras de salud se presentan también diferencias en la elección de las carreras Compeán et al. (2006), encontraron en este campo motivos asociados con la masculinidad en el caso de medicina mientras que las enfermeras refirieron más motivos vinculados al rol femenino como el interés por cuidar de los demás. En México se ha observado un aumento importante de la matrícula femenina en la educación superior, aun en aquellas carreras consideradas tradicionalmente para hombres como es el caso de la ingeniería donde la irrupción de las mujeres ha sido la más elevada en comparación con las otras áreas y las carreras de mayor preferencia femenina (Razo, 2008).

El proceso educativo es de suma importancia para el desarrollo personal, académico y profesional de un individuo; el verdadero objetivo de la educación es lograr la equidad de género, lo que significa erradicar todas las formas de manifestación del sexismo en los procesos educativos, (Solís 2016, citado en Tamayo et al, 2020).

En los primeros días de la segunda ola del movimiento feminista, las mujeres comenzaron a trabajar juntas en temas de equidad en economía y educación y desarrollaron estrategias para cabildear por la legislación y las regulaciones administrativas que afectan a las mujeres y comenzaron a construir redes políticas a través de las cuales podrían lograr reformas, al hacerlo, moldearon o incluso transformaron sus propias carreras, (Daniels, 1991).

Al iniciar el siglo $\mathrm{XX}$, las mujeres llegan a constituir la gran mayoría del personal docente de las escuelas, por un lado, debido a que se abrieron espacios educativos para que las niñas estudiaran en igual cantidad que sus compañeros, lo que repercutió en espacios laborales para las maestrasy por otro lado los hombres no se interesaron por estudiar la carrera normalista (González, 2009).

Es así, como actualmente los hombres también son minorías en las carreras relacionadas con la Educación Infantil, las restricciones estructurales dan forma y limitan las carreras, una de esas restricciones es la diferenciación de género que impregna la ocupación docente, los planes de carrera son provisionales y cambiantes, aunque no exclusivamente para las mujeres, Acker (1992). Para (Villa, 2000), el ingreso de varones en las licenciaturas de Educación Infantil ha generado que su presencia sea admitida mediante un proceso de objetivación a partir de la imagen de familia, mientras ellas se convierten 
en madres simbólicas, ellos se transforman en padres sustitutos, manteniendo un orden establecido. Este espacio podrá liberar tanto a hombres como a mujeres de esquemas culturales establecidos socialmente, en la medida en que se compartan las tareas y esto permita revalorar la profesión socialmente. Los hombres que ingresan en estas licenciaturas compartirán con las mujeres esta profesión en la medida en que desarrollen la sensibilidad hacia los otros. Para (García et al, 2015), las autoridades educativas, madres y padres, reforzaron el estereotipo de la masculinidad dominante que caracteriza esos atributos que resaltan la razón, la fuerza, la agresividad y la heterosexualidad, dudando de la capacidad de los hombres para servir como maestros en el nivel preescolar. Los argumentos muestran que, por razones de sexo, las mujeres son naturalmente aptas para convertirse en maestras porque tienen cualidades femeninas relacionadas con la ternura, la fragilidad y la maternidad.

Villanueva \& Ramírez (2015), examinaron las posibles causas de la escasa población masculina en la formación profesional docente en nivel preescolar, los hallazgos revelaron que la ideología imperante entre la comunidad que participa, guarda relación con los pensamientos y esquemas culturales de una sociedad que se ha encargado de infiltrar de generación en generación una identidad que determina las expectativas académicas y laborales en razón del género de las personas, con lo cual se ha conformado espacios simbólicamente sexistas en la formación y ejercicio profesionales. Así mismo, las barreras culturales y actitudinales, que tienen que ver con comportamientos discriminatorios de las familias o incluso del equipo docente son parte de las problemáticas que de alguna manera detienen la inclusión educativa, (Guevara \& Vélez, 2020)

Los anteriores estudios han mostrado como las diferentes carreras universitarias están también determinadas por el género, sin embargo, nos preguntamos si realmente esta diferencia de género influye también en el desempeño académico de los estudiantes, por tal motivo el objetivo del presente estudio es establecer si el género es un factor significativo en el rendimiento de los estudiantes en Educación Infantil.

\section{METODOLOGÍA}

Se explora cuantitativamente la base de datos de la Universidad Surcolombiana, desde el año 2013 hasta el 2018. Una vez efectuada la captura de datos, se aplicó el "Unpaired t test results hombres/mujeres ( $P$ value and statistical significance)" para conocer el impacto de la variable género en el rendimiento académico.

\subsection{DISEÑO Y PARTICIPANTES}

Participaron 667 (658 mujeres y 9 hombres) estudiantes de Educación Infantil de la facultad de Educación de la Universidad Surcolombiana.

\subsection{Análisis de datos}

Tabla 1 Distribución de la Calificación último semestre cursado

\begin{tabular}{|c|c|c|c|c|c|c|c|}
\hline & rango & $0<=\mathrm{x}<1,0$ & $1,0<=\mathrm{x}<2,0$ & $2,0<=\mathrm{x}<3,0$ & $3,0<=\mathrm{x}<4,0$ & $4,0<=\mathrm{x}<5,0$ & Total \\
\hline \multirow{2}{*}{ Mujeres } & $\mathrm{N}$ & 29 & 33 & 42 & 249 & 305 & 658 \\
\cline { 2 - 8 } & $\%$ & 4 & 5 & 6 & 38 & 46 & 99 \\
\hline \multirow{2}{*}{ Hombres } & $\mathrm{N}$ & & & & 5 & 4 & 9 \\
\cline { 2 - 8 } & $\%$ & & & & 56 & 44 & 100 \\
\hline
\end{tabular}


Para elaborar la Tabla 1 Distribución de la Calificación último semestre cursado, se tomó el promedio del último semestre de los estudiantes, se puede observar que ningún estudiante hombre tiene promedio por debajo de 3,0 que es la nota aprobatoria y que la proporción en el rango de calificaciones altas, superiores a 4,0 es similar al de las mujeres, cercana a $45 \%$.
Si en lugar del último semestre, se toman los promedios ponderado del primer semestre, como lo muestra la Tabla 2 Distribución de la Calificación primer semestre, la proporción en el rango de calificaciones altas se incrementa notablemente para los hombres, siendo mayor que las mujeres en 7 puntos porcentuales.

Tabla 2 Distribución de la Calificación primer semestre

\begin{tabular}{|l|r|r|r|r|r|r|r|}
\hline & rango & $0<=\mathrm{x}<1,0$ & $1,0<=\mathrm{x}<2,0$ & $2,0<=\mathrm{x}<3,0$ & $3,0<=\mathrm{x}<4,0$ & $4,0<=\mathrm{x}<5,0$ & Total \\
\hline Mujeres & $\mathrm{N}$ & 17 & 13 & 27 & 277 & 323 & 658 \\
\hline & $\%$ & 3 & 2 & 4 & 42 & 49 & 99 \\
\hline Hombres & $\mathrm{N}$ & - & - & - & 4 & 5 & 9 \\
\hline & $\%$ & - & - & - & 44 & 56 & 100 \\
\hline
\end{tabular}

Los hombres no van atrasados en sus semestres, ni han dejado de matricularse, mientras que las mujeres tienen la distribución ilustrada en Tabla 3 Semestre sin matrícula, calculada sobre toda la población que estaba inscrita el primer semestre de 2013.

Tabla 3 Semestre sin matrícula

\begin{tabular}{|c|c|}
\hline $\begin{array}{c}\text { Semestres sin } \\
\text { matrícula }\end{array}$ & $\%$ \\
\hline 0 & 37 \\
\hline 1 & 6 \\
\hline 2 & 4 \\
\hline$>3$ & 54 \\
\hline
\end{tabular}

Se realizó la prueba t-test, para determinar si estas diferencias son estadísticamente significativas, la cual aparece en la Tabla 4. Unpaired t test results hombres/mujeres, para ello, se hicieron dos grupos los que aprobaron y todo el grupo, en ambos casos la prueba resultó negativa: no hay diferencias estadísticamente significativas. 
Tabla 4. Unpaired t test results hombres/mujeres

\begin{tabular}{|c|c|c|c|}
\hline \multirow{2}{*}{\multicolumn{2}{|c|}{$\begin{array}{l}t \text { test } \\
\text { total }=564\end{array}$}} & APROBADOS & $\begin{array}{l}\text { TODO EL } \\
\text { GRUPO }\end{array}$ \\
\hline & & total $=667$ & \\
\hline $\begin{array}{l}P \text { value and } \\
\text { statistical } \\
\text { significance: }\end{array}$ & $\begin{array}{l}\text { The two-tailed } P \text { value equals } \\
\text { By conventional criteria, this difference is } \\
\text { considered to be } \\
\text { The mean of Group One minus Group }\end{array}$ & $\begin{array}{l}0,3647 \\
\text { not statistically } \\
\text { significant }\end{array}$ & $\begin{array}{c}0,5299 \\
\text { not } \\
\text { statistically } \\
\text { significant }\end{array}$ \\
\hline $\begin{array}{l}\text { Confidence } \\
\text { interval: }\end{array}$ & $\begin{array}{l}\text { Two equals } \\
95 \% \text { confidence interval of this difference }\end{array}$ & $\begin{array}{c}1,522 \\
\text { "- } 1,774 \text { to } 4,818 "\end{array}$ & $\begin{array}{c}-2,16 \\
-8,913 \text { to } \\
4,592\end{array}$ \\
\hline \multirow{3}{*}{$\begin{array}{l}\text { Intermediate } \\
\text { values used in } \\
\text { calculations: }\end{array}$} & $t=$ & $t=0.9071$ & 0,6285 \\
\hline & $\mathrm{df}=$ & $\mathrm{df}=562$ & 665 \\
\hline & standard error of difference $=$ & 1,678 & 3,437 \\
\hline
\end{tabular}

En la Tabla 5 promedio y desviación estándar, se puede observar que el promedio de los hombres es ligeramente superior al de las mujeres y más centrado en torno a la media, ya que su desviación estándar es menor que en las mujeres, o dicho en otras palabras el rendimiento de los hombres fue menos disperso, más uniforme.

Tabla 5. Promedio y desviación estándar

\begin{tabular}{|c|c|c|c|c|}
\hline \multirow[b]{2}{*}{ Grupo } & \multicolumn{2}{|c|}{$\begin{array}{c}\text { APROBADOS } \\
\text { total }=564\end{array}$} & \multicolumn{2}{|c|}{$\begin{array}{c}\text { TODO EL GRUPO } \\
\text { total }=667\end{array}$} \\
\hline & hombres & Mujeres & hombres & mujeres \\
\hline promedio & 39 & 40 & 39 & 36 \\
\hline SD & 5 & 5 & 5 & 10 \\
\hline SEM & 2 & 0 & 2 & 0 \\
\hline $\mathrm{N}$ & 9 & 555 & 9 & 658 \\
\hline
\end{tabular}

La evolución de las calificaciones a lo largo de los semestres se muestra en la Tabla 6, en ella aparecen todos los promedios ponderados obtenidos por los estudiantes a lo largo de la carrera. 


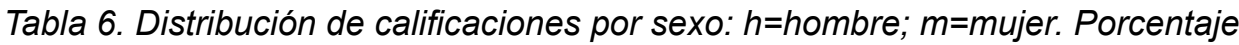

\begin{tabular}{|c|c|c|c|c|c|c|c|c|c|c|c|c|c|c|c|c|c|c|}
\hline & \multicolumn{2}{|c|}{$2014-1$} & \multicolumn{2}{|c|}{ 2014-2 } & \multicolumn{2}{|c|}{ 2015-1 } & \multicolumn{2}{|c|}{$2015-2$} & \multicolumn{2}{|c|}{ 2016-1 } & \multicolumn{2}{|c|}{ 2016-2 } & \multicolumn{2}{|c|}{$2017-1$} & \multicolumn{2}{|c|}{ 2017-2 } & \multicolumn{2}{|c|}{ 2018-1 } \\
\hline & $\mathrm{h}$ & $\mathrm{m}$ & $\mathrm{h}$ & $\mathrm{m}$ & $\mathrm{h}$ & $\mathrm{m}$ & $\mathrm{h}$ & $\mathrm{m}$ & $\mathrm{H}$ & $\mathrm{m}$ & $\mathrm{h}$ & $\mathrm{m}$ & $\mathrm{h}$ & $\mathrm{m}$ & $\mathrm{H}$ & $M$ & $\mathrm{~h}$ & $\mathrm{~m}$ \\
\hline $0<=1$ & - & 1 & - & - & - & 1 & - & 1 & - & 3 & - & 1 & - & 1 & - & 0 & - & - \\
\hline $1<=2$ & - & 3 & - & 1 & - & 2 & - & 1 & - & 2 & - & 2 & - & 1 & - & 1 & - & 0 \\
\hline $2<=3$ & - & 7 & - & 5 & - & 9 & - & 4 & - & 7 & - & 6 & - & 8 & - & 8 & - & 7 \\
\hline $3<=4$ & 100 & 40 & - & 42 & 25 & 42 & 40 & 50 & 60 & 52 & 83 & 56 & 38 & 54 & 50 & 59 & 50 & 58 \\
\hline $4<=5$ & & 49 & 100 & 53 & 75 & 46 & 60 & 43 & 40 & 36 & 17 & 35 & 63 & 37 & 50 & 31 & 50 & 34 \\
\hline
\end{tabular}

La anterior Tabla 6, se ilustra en el Gráfico 1 Evolución hombres y mujeres a lo largo de la carrera

Evolución de notas Hombres

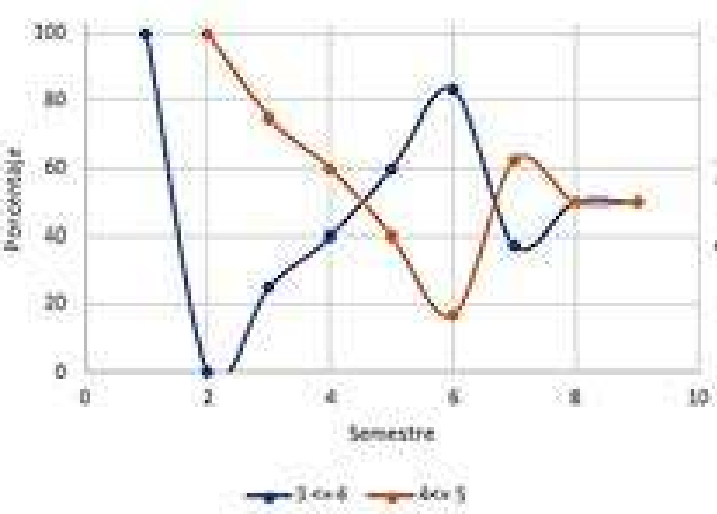

Evelución de notas Mujeres

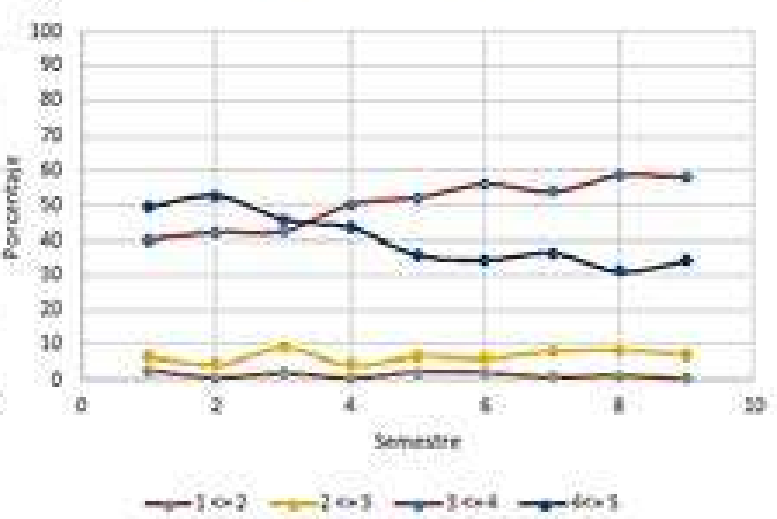

Gráfico 1 Evolución hombres y mujeres a lo largo de la carrera

En el Gráfico 1, al comienzo de la carrera, se pude observar que el número de estudiantes mujeres que obtienen notas entre 3 y 4 es mayor. Esta relación se invierte a partir del 3 semestre. En los hombres ocurre igual, pero se invierte a partir del 2 semestre y al final de la carrera se iguala al de rango superior en $50 \%$. También, se advierte en hombres que no hay estudiantes con notas menores a 3

\section{RESULTADOS Y DISCUSIÓN}

En cuanto al rendimiento de los estudiantes en Educación Infantil los resultados muestran que no hay diferencias estadísticamente significativas, aunque el promedio de los hombres es ligeramente superior al de las mujeres y más centrado en torno a la media. Por otro lado, más mujeres que hombres obtienen notas entre tres y cuatro pero esta relación se invierte a partir del tercer semestre, estos resultados concuerdan con los estudios de Francesconi \& Parey (2018) con seis cohortes de universitarios en Alemania cuyos resultados muestran que las mujeres ingresan a la universidad con calificaciones ligeramente mejores, aunque abandonan la universidad con calificaciones ligeramente más bajas. 


\section{CONCLUSIONES}

En general, puede derivarse del presente estudio que la variable género no influye en el rendimiento de los cursos de Educación Infantil.

Es importante que las universidades aumenten el número de hombres en su programa de Educación Infantil, pues no se requieren cambios ni diseño de currículos especiales y, por otra parte, la universidad y la sociedad se están privando de las capacidades de los hombres en estos campos.

\section{REFERENCIAS BIBLIOGRÁFICAS}

Acker, S. (1992). Special Series on Girls and Women in Education: Creating Careers: Women Teachers at Work. Curriculum inquiry, 22(2), 141-163.

Albertín Lasaosa, A. M., \& Zufiaurre Goicoechea, B. (2005). La formación del profesorado de Educación Infantil. Una trayectoria desde la LGE hasta la LOE. Universidad Pública de Navarra/Nafarroako Unibertsitate Publikoa.

Brenøe, A. A., \& Lundberg, S. (2018). Gender gaps in the effects of childhood family environment: Do they persist into adulthood?. European Economic Review, 109, 42-62.

Burton, D. J., \& Darcy, R. (1985). Careers of Men and Women in the Profession: the 1970-1975 Cohort. Western Political Quarterly, 38(1), 132-147. https://www. jstor.org/stable/448288

Cozzi, G., Francesconi, M., Lundberg, S., Mantovan, N., \& Sauer, R. M. (2018). Advancing the economics of gender: New insights and a roadmap for the future. European Economic Review, 109(C), 1-8.)
Compeán Dardón, M., Verde Flota, E., Gallardo Hernández, G., Tamez González, S., Delgado Sánchez, G., \& OrtizHernández, L. (2006). Diferencias entre hombres y mujeres respecto a la elección de carreras relacionadas con atención a la salud. La ventana. Revista de estudios de género, 3(24), 204-228.

Daniels,A. K. (1991). Careers infeminism. Gender \& Society, 5(4), 583-607.

Ferber, M., \& Huber, J. (1979). Husbands, wives, and careers. Journal of Marriage and the Family, 315-325.

Francesconi, M., \& Parey, M. (2018). Early gender gaps among university graduates. European Economic Review, 109, 63-82.

González Jiménez, R. M. (2009). De cómo y por qué las maestras llegaron a ser mayoría en las escuelas primarias de México, Distrito Federal (finales del siglo XIX y principios del $\mathrm{XX}$ ): un estudio de género. Revista mexicana de investigación educativa, 14(42), 747785.

Guevara Salazar LX, Vélez Villaquirán (2020) Primeras aproximaciones sobre la realidad de la inclusión en la educación en Colombia. redipe 9(3):85-100. Disponible en: https://revista.redipe.org/ index.php/1/article/view/933

García Villanueva, J., Ávila Rodríguez, D., Vargas Pérez, M. K., \& Hernández Ramírez, C. I. (2015). Acerca de la feminización de profesiones. Caso: la docencia en preescolar en la Cuidad de México. La ventana. Revista de estudios de género, 5(42), 129-151. 
Kauhanen, A., \& Napari, S. (2011). Gender differences in careers. Annals of Economics and Statistics. (117), 61-88.

Luukkonen-Gronow, T. (1987). University career opportunities for women in Finland in the 1980s. Acta Sociologica, 30(2), 193-206. https://www.jstor.org/stable/4194677

Razo Godínez, M. L. (2008). La inserción de las mujeres en las carreras de ingeniería y tecnología. Perfiles educativos, 30(121), 63-96.

Skachkova, P. (2007). Academic careers of immigrant women professors in the US. Higher Education, 53(6), 697-738.

Tamayo Arguello V, Reyes Guillén I, Fonseca Córdoba S. (2020) Educación media superior: una vía de empoderamiento para la mujer indígena. redipe, 9(2):16372. Disponible en: https://revista.redipe. org/index.php/1/article/view/918

Villa, M.P. (2000). Espacios eidentidades: ingreso de profesores a preescolar. Revista de estudios de género. La ventana, (12), 147-176.

Villanueva, J. G., \& Ramírez, C. I. H. (2015). La formación del profesorado de nivel preescolar: un campo que excluye a los hombres en la ciudad de México. Entreciencias: Diálogos en la sociedad del conocimiento, 3(7), 241251. 